\title{
PRIMARY HYPERPARATHYROIDISM AS AN UNUSUAL CAUSE OF CHRONIC PANCREATITIS: A CASE REPORT
}

T. Savitri1 ${ }^{1}$ B. Shwetha², D. Sai Raghavendra ${ }^{3}$, P. Radha4

\section{HOW TO CITE THIS ARTICLE:}

T. Savitri, B. Swetha, D. Sai Raghavendra, P. Radha. "Primary Hyperparathyroidism as an Unusual Cause of Chronic Pancreatitis: A Case Report". Journal of Evolution of Medical and Dental Sciences 2015; Vol. 4, Issue 35, April 30; Page: 6158-6161, DOI: 10.14260/jemds/2015/897

ABSTRACT: Chronic pancreatitis is a relatively rare disorder occurring in 1.6 to 13 per 100,000 population. This is a progressive disease with persistent inflammation leading to damage and/or destruction of the anatomy and functioning of pancreas. The etiology is multifactorial. Alcohol consumption is implicated in $73 \%$ of cases. Primary hyperparathyroidism is an unusual cause and literature is limited on this interesting association. Hypercalcemia is thought to be the mechanism by which hyperparathyroidism causes chronic pancreatitis. In this paper, we discuss a case of an 18 year old female patient presented with prolonged dull unremitting upper abdominal pain since 3 years with hypercalcemia as cause due to parathyroid adenoma.

KEYWORDS: Primary Hyperparathyroidism, Chronic Pancreatitis.

INTRODUCTION: Chronic pancreatitis is characterized by continuous inflammatory changes of pancreas leading to irreversible morphological changes and /or permanent loss of function.(1)

The common causes of chronic pancreatitis include chronic alcohol abuse, idiopathic pancreatitis, hyperparathyroidism, hereditary pancreatitis, tropical pancreatitis, autoimmune pancreatitis and hypertriglyceridemia.(1)

Primary hyperparathyroidism is a rare cause of both acute and chronic pancreatitis.1-4\%.(1) Chronic pancreatitis occur in cases of untreated hyperparathyroidism. Hypercalcemia due to hyperparathyroidism causes increase in calcium secretion of pancreas.

Many case reports(2-3-4) and case series ${ }^{(2-4)}$ proved the cause and association of primary hyperparathyroidism and chronic pancreatitis.

CASE REPORT: An 18 years old adolescent female presented with recurrent acute episodes over constant dull upper abdominal pain since 3 years. Her serum amylase and lipase levels were elevated. Ultrasound of abdomen revealed an enlarged hypoechoic pancreas with coarse calcifications all over parenchyma. There is uneven dilatation of main pancreatic duct up to $6 \mathrm{~mm}$ with few intraductal calculi, hyperechogenic gall bladder sludge and bilateral bulky hyperechogenic kidneys.

Contrast enhanced CT of abdomen revealed bulky pancreas with coarse intra pancreatic calcifications all over pancreas and dilated main pancreatic duct up to $6 \mathrm{~mm}$ with few intra ductal calculi and hyperdense sludge in gall bladder. Bilateral bulky kidneys with few tiny calculi.

MRCP showed irregularly dilated main pancreatic duct and few side branch dilatation.

In view of calcific pancreatitis and renal stones, hypercalcemia is suspected and serum calcium were done, which is slightly elevated up to $13 \mathrm{mg} / \mathrm{dl}$. On evaluating causes for hypercalcemia, hyperparathyroidism is suspected.

Thereby, on ultrasonogaphy of neck revealed well defined homogenously hypoechoic nodule located inferior to left lobe of thyroid suggestive of left parathyroid adenoma as a cause for primary 


\section{CASE REPORT}

hyperparathyroidism. Which is then confirmed by elevated serum paratharmone levels to 132 $\mathrm{ng} / \mathrm{ml}$ ? Later parathyroidectomy was done for the patient which is confirmed histopathologically as parathyroid adenoma.

On follow up after 6 months, there is decline in frequency and severity of acute episodes. Serum calcium levels and serum paratharmone levels gradually returned to normal.

DISCUSSION: Chronic pancreatitis is characterized by the inflammatory changes of pancreas involving following: fibrosis, calcification, ductal inflammation and pancreatic stone formation. Finally, endocrine and exocrine functional impairment results from the irreversible pancreatic injury. This produces constant dull pain, weight loss, steatorrhea, glucose intolerance and weight loss.

Alcohol constitutes most common cause in western world. The incidence of chronic pancreatitis has quadrupled risk per gm of alcohol per day over 6 to 12 years. In tropical countries, tropical pancreatitis is commonest non-alcholic etiology. Genetic diseases (e.g., cystic fibrosis) and anatomic defects predominate in children. Pancreas divisum is commonest cause among congenital anomalies.

The TIGAR-O (Toxic-metabolic, Idiopathic, Genetic, Autoimmune, Recurrent and severe acute pancreatitis, Obstructive) classification system is based on risk factors for chronic pancreatitis.[5]

Primary hyperparathyroidism (PHPT) is a rare cause of chronic pancreatitis. Presence of normal or increased serum calcium in the setting of pancreatitis raises the suspicion of primary hypercalcemic state as the underlying cause for pancreatitis.

Pancreatitis related to PHPT was first reported over 60 years ago.[5] The healing of pancreatitis after parathyroidectomy favours this hypothesis.

Data from the west suggests an association in upto 7\% of cases.(6) Centers from India have reported a prevalence of up to $15.2 \% .^{[7]}$ The largest study from India is a recent prospective, nationwide study of 1, 086 subjects of chronic pancreatitis carried out by The Indian Pancreatitis Study Group. The results were as follows: Idiopathic pancreatitis was the most common form of pancreatitis (60.2\%) and alcoholic chronic pancreatitis accounted for about a third of the cases (38.7\%). Using well-defined criteria, only 39(3.8\%) cases could be labeled as 'tropical pancreatitis. The rest (1. 1\%) had rare risk factors, including pancreas divisum $(0.4 \%)$ and hyperparathyroidism $(0.2 \%) ;(8)$ in addition, one patient each $(0.1 \%)$ had agenesis of the pancreas, autoimmune pancreatitis, hypertriglyceridemia-related pancreatitis, trauma and microlithiasis.

Chronic pancreatitis due to PHPT has important characteristics in its biochemical and clinical manifestations. When compared to alcohol-related chronic pancreatitis and idiopathic chronic pancreatitis, the incidence of renal colic, nephrolithiasis, and nephrocalcinosis were significantly more common in patients with chronic pancreatitis due to PHPT.[9] Similarly, bone disease, palpable neck nodule, and psychiatric abnormalities were statistically more common in the latter group. The complications of CP such as steatorrhea, diabetes mellitus, pancreatic calcification, and pancreatic pseudocyst were not statistically different across the three groups.

When patients of chronic pancreatitis due to PHPT were compared with PHPT patients without chronic pancreatitis, renal colic, nephrolithiasis, steatorrea, and diabetes are common in patients with chronic pancreatitis. The mean corrected calcium and mean iPTH levels were significantly higher in patients of chronic pancreatitis due to PHPT, while other biochemical parameters such as serum amylase, serum alkaline phosphatase (ALP), blood sugar, and triglyceride 
levels were not different from patients with alcohol-related chronic pancreatitis and idiopathic chronic pancreatitis.[10]

The issue of the relationship between primary hyperparathyroidism and pancreatitis and its mechanism needs to be clearly resolved. The mechanism of development of pancreatic disease in PHPT is correlated to the hypercalcaemia.[11] Hypercalcaemia per se, in addition to being an independent risk for the precipitation of pancreatic cellular injury, could also augment pancreatic disease in patients with ongoing pancreatic injury because of other causes. The exact cellular mechanism by which hyper-calcaemia causes pancreatic injury in PHPT remains to be elucidated..11]

\section{REFERENCES:}

1. Etemad B, Whitcomb DC. Chronic pancreatitis: diagnosis, classification, and new genetic developments. Gastroenterology 2001; 120: 682-707.

2. Carnaille B, Oudar C, Pattou F, Combemale F, Rocha J, Proye C. Pancreatitis and primary hyperparathyroidism: forty cases. Aust. N. Z. J. Surg. 1998; 68: 117-19.

3. Niederle B, Roka R, Woloszczuk W, Klaushofer K, Kovrik J, Schernthaner G. Successful parathyroidectomy in primary hyperparathyroidism: a clinical follow-up study of 212 consecutive patients. Surgery 1987; 102: 903-9.

4. Herskovic T, Keating FR, Ross JB. Coexistent pancreatitis and hyperparathyroidism: observation on 15 cases. Gastroenterology 1967; 52: 1093.

5. Nair RJ. Chronic Pancreatitis. Am Fam Physician2007; 76:1679-1688.

6. Bess MA. Hyperparathyroidism and Pancreatitis. Chance or a Causal Association? JAMA 1980; 243: 246247.

7. Balakrishnan V. Chronic Pancreatitis. A Prospective Nationwide Study of 1, 086 Subjects from India. JOP. J Pancreas (Online) 2008; 9: 593-600.

8. Cope O. Pancreatitis, a diagnostic clue to hyperparathyroidism. Ann Surg 1957; 145: 857-863.

9. Jacob JJ. Does hyperparathyroidism cause pancreatitis? A South Indian experience and a review of published work. ANZ J Surg 2006; 76: 740-744.

10. Bhadada SK. Chronic pancreatitis in primary hyperparathyroidism: Comparison with alcoholic and idiopathic chronic pancreatitis. J Gastroenterol Hepatol 2008; 23: 959-964.

11. Carnail B. Pancreatitis and primary hyperparathyroidism: Forty cases. Aust N Z J Surg 1998; 68: 117-119. 


\section{CASE REPORT}

\section{AUTHORS:}

1. T. Savitri

2. B. Shwetha

3. D. Sai Raghavendra

4. P. Radha

\section{PARTICULARS OF CONTRIBUTORS:}

1. Assistant Professor, Department of Radio diagnosis, AMC, Visakhapatnam.

2. $2^{\text {nd }}$ Year Post Graduate, Department of Radio diagnosis, AMC, Visakhapatnam.

3. $3^{\text {rd }}$ Year Post Graduate, Department of Radio diagnosis, AMC, Visakhapatnam.

FINANCIAL OR OTHER

COMPETING INTERESTS: None
4. $3^{\text {rd }}$ Year Post Graduate, Department of Radio diagnosis, KMC, Manipal.

\section{NAME ADDRESS EMAIL ID OF THE CORRESPONDING AUTHOR:}

Dr. T. Savitri,

House No.50-102-6, Amulya, Near Krishna Mandir, Seethamadara Ne,

Visakhapatnam-530013.

E-mail: thanukulasavitri@gmail.com

Date of Submission: 29/03/2015.

Date of Peer Review: 30/03/2015.

Date of Acceptance: 23/04/2015.

Date of Publishing: 30/04/2015. 